\title{
AVALIAÇÃO DO ÍNDICE DE SATISFAÇÃO E QUALIDADE DE VIDA DOS PACIENTES ATENDIDOS NO SETOR DE FISIOTERAPIA CARDIORRESPIRATÓRIA NA CLÍNICA DA FACULDADE ANHANGUERA DE TAUBATÉ
}

\author{
EVALUATION OF SATISFACTION RATE AND QUALITY OF LIFE OF PATIENTS TREATED IN THE \\ CARDIORESPIRATORY PHYSIOTHERAPY SECTOR IN THE CLINIC OF \\ THE ANHANGUERA COLLEGE OF TAUBATÉ
}

\author{
Liliane de Faria Marcon \\ Curso de Fisioterapia \\ Faculdade Anhaguera de Taubaté \\ e São José dos Campos

\section{Bruna Stefanie Lopes da Silva \\ Juliana de Oliveira Ribeiro} \\ Curso de Fisioterapia \\ Faculdade Anhaguera de Taubaté
}

\author{
Contato \\ Liliane de Faria Marcon \\ Av. Dr. José Olegário de Barros, 46/58 \\ Vila Nossa Senhora das Graças \\ Taubaté-SP \\ CEP: $12060-400$ \\ E-mail: liliane.marcon@usp.br
}

\section{RESUMO}

Introdução: A Reabilitação cardiopulmonar e metabólica é um programa multiprofissional que visa ao desenvolvimento e à manutenção do condicionamento físico, social e psicológico, incluindo treinamento físico, educação, fisioterapia funcional e respiratória e apoio psicossocial. É uma forma de melhorar a terapia padrão, controlando e aliviando sintomas e aperfeiçoando sua capacidade funcional. A avaliação dos resultados obtidos com os serviços prestados é muito importante para estabelecer novas metas e melhorias, ainda mais em serviços de clínica escola. Objetivo: Analisou-se o índice de satisfação e qualidade de vida dos pacientes tratados no setor de fisioterapia cardiorrespiratória da Clínica Escola de Taubaté. Métodos: Foram aplicados dois questionários aos voluntários: um semiestruturado, que avaliou a satisfação dos pacientes, e o SF-36, que avaliou qualidade de vida. Resultados: Dos participantes, $52 \%$ eram do sexo masculino, e os diagnósticos clínicos mais presentes foram hipertensão arterial sistêmica e doença pulmonar obstrutiva crônica. A satisfação com o serviço prestado foi avaliado como "Muito Bom" e "Bom" em $100 \%$ dos casos. Das médias de qualidade de vida, os aspectos sociais e de condição geral de saúde foram os mais bem avaliados, no entanto, limitações por aspectos emocionais e vitalidade foram os que menos pontuaram. Conclusão: $O$ trabalho demonstrou que o serviço oferecido foi avaliado como com alta qualidade pelos usuários; ademais, verificou-se que o impacto social foi o de maior influência.

Palavras-chave: Satisfação do Paciente, Reabilitação, Exercícios Respiratórios. 


\section{ABSTRACT}

Introduction: Cardiopulmonary and metabolic rehabilitation is a multiprofessional program that aims at the development and maintenance of physical, social and psychological conditioning, including physical training, education, functional and respiratory physiotherapy and psychosocial support. It is a way to improve standard therapy by controlling and alleviating symptoms and improving functional capacity. The evaluation of the results obtained with the services provided is very important to establish new goals and betterments, specially in school clinic services. Objective: To analyze the satisfaction index and quality of life of the patients treated in the cardiorespiratory physiotherapy sector of the Clinic School of Taubaté. Methods: Two questionnaires were conducted with the volunteers, a semi-structured questionnaire that evaluated patient satisfaction and the SF-36 evaluating quality of life. Results: Of all participants, 52\% were male and the most frequent clinical diagnoses were systemic arterial hypertension and chronic obstructive pulmonary disease. Satisfaction with the service provided was evaluated as "very good" and "good" in $100 \%$ of cases. Of the averages of quality of life, the social aspects and general health condition were the best evaluated and limitations due to emotional aspects and vitality were the ones that scored the least. Conclusion: the study demonstrated that the service offered was rated as high quality by the users and it was verified that the social impact was the one with the greatest influence.

Keywords: Patient Satisfaction. Rehabilitation. Breathing Exercises.

\section{INTRODUÇÃO}

A fisioterapia tem se tornado uma ferramenta fundamental na vida de muitos pacientes, contribuindo significativamente para um melhor prognósti$\mathrm{CO}^{1-2}$. Por meio dela, muitos pacientes têm encontrado um meio para uma melhora em sua qualidade de vida, em seus sintomas, para ganhar condicionamento físico e, assim, voltar a suas atividades, que até então não mais realizavam devido a suas limitações ${ }^{3-5}$.

No setor de Fisioterapia Cardiorrespiratória, a Reabilitação cardiopulmonar e metabólica (RCPM) é uma forma de melhorar a terapia padrão. A reabilitação inclui o treinamento físico, educação, fisioterapia funcional e respiratória, bem como apoio psicossocial, aperfeiçoando a capacidade funcional do paciente, gerando condicionamento físico, alívio de sintomas e maior espaçamento entre crises, além de trazer benefícios à vida desses pacientes, para realizar desde as atividades mais simples do dia a dia até atividades esportivas ${ }^{6-8}$.

Devido aos maus hábitos de vida, a incidência de doenças crônicas vem aumentando no Brasil e no mundo. Em 1993, a Organização Mundial da Saúde (OMS) já recomendava que portadores de doenças cardiovasculares devessem ser encaminhados para programas de reabilitação, sendo considerado incompleto o tratamento realizado apenas com medicamentos ${ }^{9-10}$

A análise de satisfação do cliente nesses estabelecimentos deve ser vista de forma multidimensional, ou seja, em todos os aspectos da assistência ${ }^{11}$; incluindo desde o acesso ao serviço, como a estrutura física, acolhimento, confiança, a relação entre profissional-paciente, até o ponto de maior importância: o nível de melhoria no condicionamento físico e na qualidade de vida dos pacientes atendidos no setor ${ }^{12}$.

Assim, o objetivo do estudo foi avaliar a satisfação dos pacientes atendidos no setor de fisioterapia cardiorrespiratória da Clínica Escola de Fisioterapia da Faculdade Anhanguera de Taubaté, levantar os pontos de maior e menor pontuação do índice da qualidade do serviço prestado e identificar as áreas de menor e maior pontuação no quesito de qualidade de vida.

\section{MÉTODO}

ए ste artigo é um estudo do tipo observacional/ descritivo, transversal, quantitativo e qualitativo, com variáveis independentes, cujos dados foram obtidos por meio de amostra de conveniência.

A pesquisa foi realizada com 27 pacientes do setor de fisioterapia cardiorrespiratória, da Clínica Escola da Faculdade Anhanguera de Taubaté - Unidade Vila das Graças, com o parecer n 1.090 .063 de aprovação do Comitê de Ética e Pesquisa (CEP).

Como critérios de inclusão, foram definidos: usuários de ambos os sexos, com idade acima de 18 anos. Foram excluídos os indivíduos com deficit cognitivo e pacientes com menos de três meses de tratamento fisioterapêutico no setor.

Os voluntários incluídos receberam dois questionários para que pudessem ser respondidos em sua residência, com o objetivo de não interferir na sessão de fisioterapia nem se sentirem pressionados ao respondê-los.

O questionário semiestruturado de satisfação e qualidade do serviço ${ }^{11}$ dispunha da identificação do paciente e de 17 questões alternativas, sendo utilizadas em algumas delas respostas Sim, Não e Indefere, e em outras, a escala tipo likert que classificava 
em Muito Bom, Bom, Regular, Ruim e Muito Ruim. As perguntas foram: 1 . Como você avalia o atendimento prestado na recepção da Clínica de Fisioterapia?; 2. Você sente-se à vontade em ser atendido por acadêmicos?; 3. Esta foi sua primeira experiência com a fisioterapia?; 4. Você tem expectativas de melhora do seu quadro, mesmo sendo submetido a atendimento por acadêmicos?; 5. Quanto ao esclarecimento de suas dúvidas pelo acadêmico; 6. Quanto à atenção dada pelo acadêmico a suas queixas; 7. Quanto à segurança transmitida pelo acadêmico durante o tratamento; 8. Como é a sua relação com o acadêmico responsável por seu tratamento?; 9. Você acredita que a presença dos professores responsáveis nos atendimentos altera o modo de atendimento do acadêmico?; 10. Você está satisfeito com a presença dos professores responsáveis pelos acadêmicos?; 11. Os atendimentos são realizados de forma pontual?; 12. Como você considera os recursos disponíveis para tratamento?; 13. Quanto à limpeza, higiene e segurança dos equipamentos/materiais utilizados pelo fisioterapeuta, como você os considera?; 14. Os acadêmicos, professores e funcionários vestem-se de maneira adequada para o serviço que prestam?; 15. Apresentou melhora com o tratamento fisioterapêutico?; 16. Você retornaria para esta unidade se precisasse novamente de fisioterapia?; 17 . Você recomendaria este serviço a familiares e amigos?
O questionário de qualidade de vida relacionado com a saúde utilizado foi o Medical Outcomes Study 36-Item Short-Form Health Survey (SF-36), que foi traduzido e adaptado para a língua portuguesa ${ }^{13}$. Ele é composto por 36 perguntas que avaliam a percepção da doença pelo ponto de vista do próprio paciente. Dos 36 itens, 35 encontram-se dentro de 8 dimensões de saúde, que são: Capacidade Funcional, Aspecto Físico, Dor, Estado Geral de Saúde, Vitalidade, Aspectos Sociais, Aspectos Emocionais e Saúde Mental. Para cada dimensão, os itens são pontuados, agrupados e transformados em uma escala de zero (pior estado de saúde) a 100 (melhor estado de saúde).

Após uma semana da entrega, os questionários foram recolhidos.

Os dados são apresentados por meio de análise descritivas, médias e desvio padrão para variáveis contínuas.

\section{RESULTADOS}

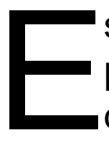
ste trabalho foi realizado com uma amostra de 27 pacientes, sendo $52 \%$ do sexo masculino e $48 \%$ do sexo feminino, com idade de 50,48 $\pm 27,16$.

As principais patologias atendidas no momento foram a hipertensão arterial sistêmica (HAS) (18\%) e a doença pulmonar obstrutiva crônica (DPOC) (15\%) (Gráfico 1).

Gráfico 1. Porcentagem de pacientes por patologias

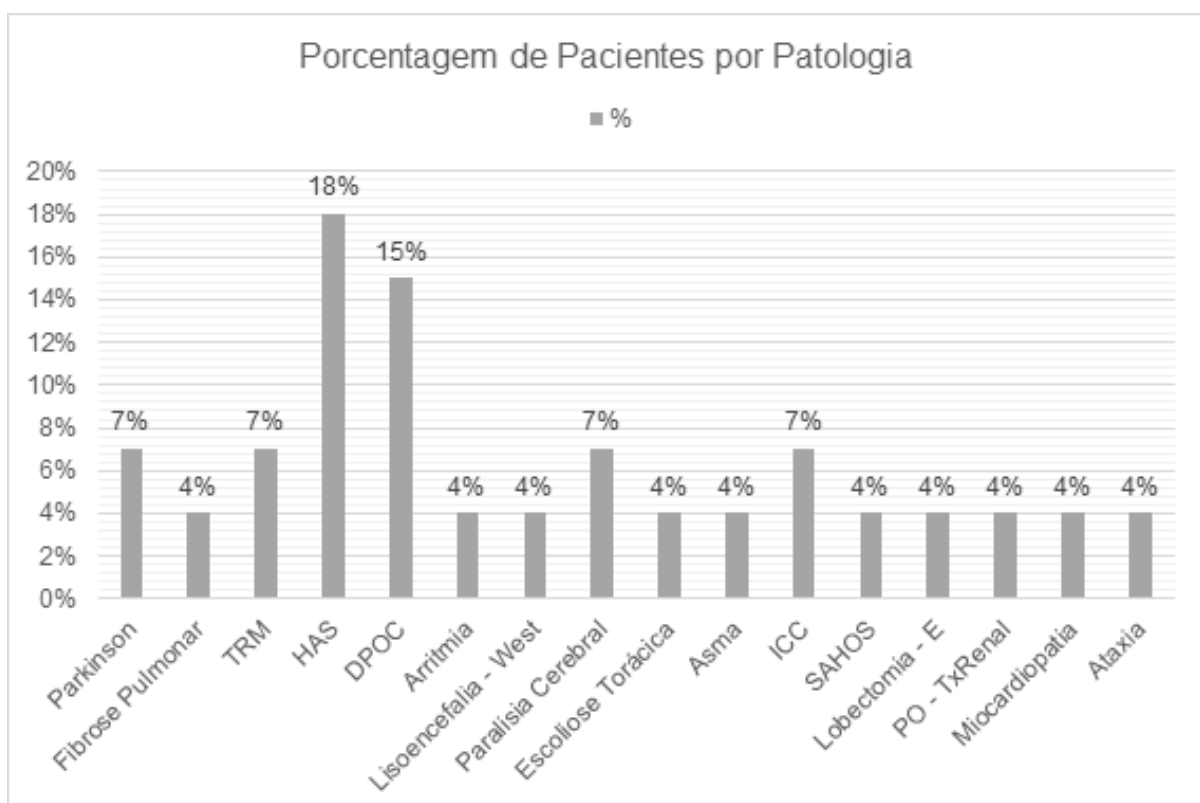


Por meio da aplicação do questionário de satisfação e qualidade de serviços prestados, foram observados os seguintes resultados:

- Como o paciente avalia o atendimento prestado na recepção da Clínica de Fisioterapia? - 78\% responderam ser Muito Bom, $22 \%$ responderam ser Bom.

- Paciente se sente à vontade sendo atendido por acadêmicos? - $100 \%$ responderam Sim.

- Foi sua primeira experiência com a fisioterapia? - 55,5\% responderam que Sim, 44,5\% responderam que Não.

- Esclarecimento de dúvidas pelo fisioterapeuta - 80,5\% responderam ser Muito Bom, 19,5\% responderam ser Bom.

- A segurança transmitida pelo fisioterapeuta durante o tratamento $-81,5 \%$ responderam ser Muito Bom, $18,5 \%$ responderam ser Bom.

- Relação do paciente com o acadêmico responsável - 81,5\% responderam ser Muito Bom, 18,5\% responderam ser Bom.

- Está satisfeito (a) com a presença dos professores responsáveis pelos acadêmicos ou acredita que eles poderiam ser mais presentes durante as sessões? - $100 \%$ responderam estar satisfeito.

- Quanto aos recursos disponíveis para tratamento (apareIhos, entre outros) - 63,5\% responderam ser Muito Bom, 33,5\% responderam ser Bom e 3,5 responderam como Regular.

- Limpeza, higiene e segurança dos equipamentos/materiais utilizados pelo fisioterapeuta $-74 \%$ responderam como Muito bom, $26 \%$ responderam ser Bom.

- Você retornaria para esta unidade se precisasse novamente de fisioterapia? $-100 \%$ dos pacientes responderam que sim (Gráfico 2).

Gráfico 2. Satisfação e qualidade dos serviços prestados

\section{Satisfação e Qualidade de Serviços Prestados}

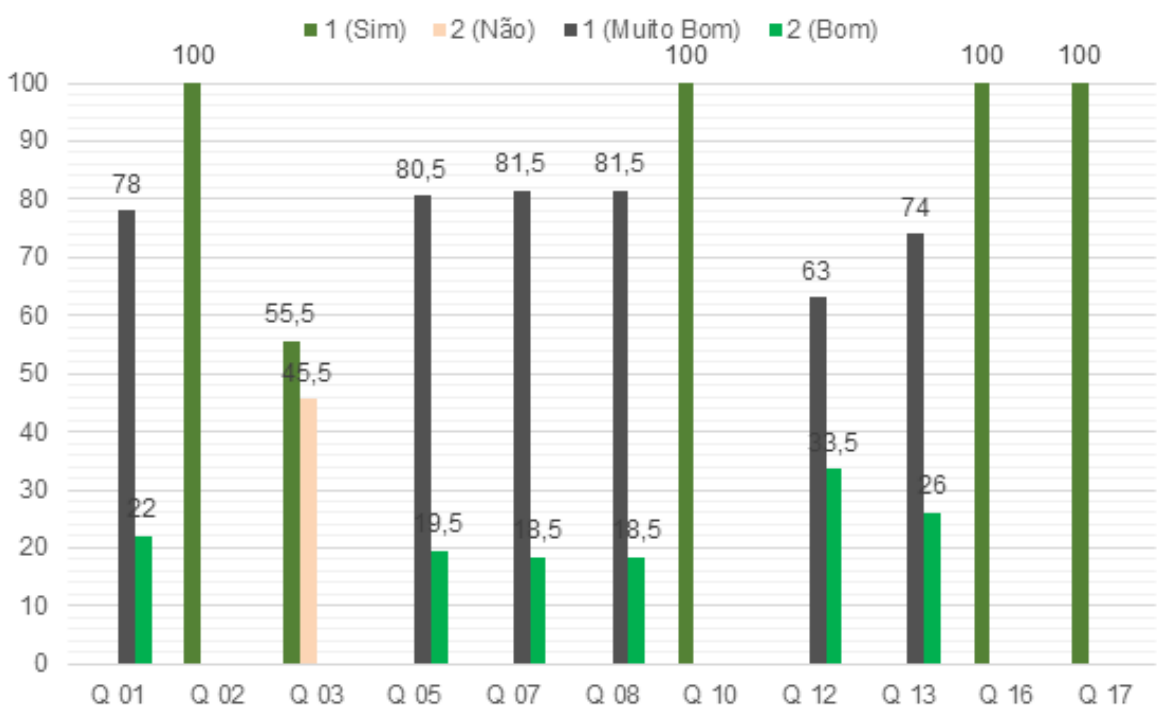


Na questão de classificação da qualidade de vida, o escore geral encontrado foi de $112 \pm 16,89$. Com o Cálculo dos Escores do questionário SF-36, foram observados os seguintes resultados:

- Capacidade funcional com média de 55,71 $\pm 31,79$, limitação por aspectos físicos com média $57,14 \pm 40,42$, dor com média $60,72 \pm 24,82$, estado geral de saúde com $73,5 \pm 14,45$, vitalidade com $50,68 \pm 10,26$, aspectos sociais com média 76,70 $\pm 23,24$, limitação por aspectos emocionais com 48,46 $\pm 42,07$ e saúde mental com média de 54,54 \pm 14,47 (Gráfico 3).

Gráfico 3. Resultados do questionário SF-36

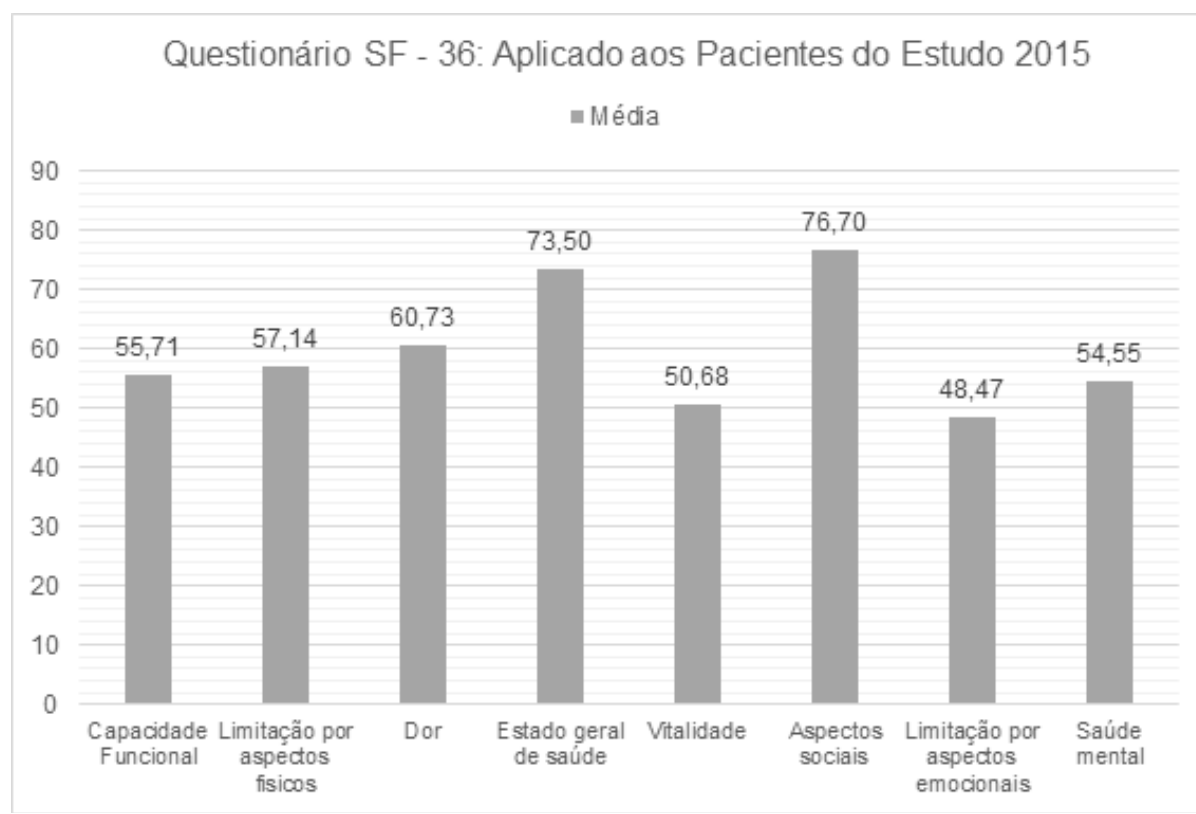

\section{DISCUSSÃO}

E ste estudo teve como principal objetivo avaliar a satisfação dos usuários do serviço de fisioterapia cardiorrespiratória de uma clínica escola e identificar os quesitos que mais interferem na qualidade de vida dos usuários.

A maior participação de pacientes do sexo masculino apresenta relação direta com o maior acometimento deles por doenças cardiovasculares. Esses dados também foram sustentados pelo trabalho de Silva et al. ${ }^{14}$, em que dos 16 pacientes avaliados, $62 \%$ eram homens. A esse fato, pode-se atribuir a maior mortalidade por doenças cardiovascular em homens, contribuindo para a busca e permanência do tratamento fisioterapêutico.

$\mathrm{Na}$ avaliação da qualidade do serviço prestado, verifica-se que os níveis de satisfação do paciente ti- veram alto conceito em todos os quesitos avaliados. No entanto, em três pontos, a totalidade dos avaliados respondeu positivamente, sendo estes quanto à segurança e relação com o acadêmico, satisfação com a presença de um supervisor e retorno à instituição. O graduando em fisioterapia é estimulado a ter uma forma crítica e reflexiva desde os primeiros anos de seu curso, trazendo para o período do estágio obrigatório esses princípios éticos e morais que regem a profissão e refletem de maneira direta no modo de tratamento de seus pacientes.

Moreira et al. ${ }^{11}$ afirmam que o conceito da qualidade da interação do paciente com seu fisioterapeuta, especialmente quando diz respeito à troca de informação, é um forte indicador da satisfação, assegurando sua participação no tratamento. Demonstraram também que a segurança transmitida pelo fisioterapeuta à comunicação e à continuidade 
da assistência foi o principal indicador da satisfação expressada pelos pacientes estudados. Os autores também afirmam que é fundamental ao tratamento uma boa relação entre o terapeuta e seu paciente, sendo de grande importância a criação dessa aliança terapêutica para o estabelecimento de uma relação de confiança e para obter melhores resultados no tratamento. Johnston e Clark $^{15}$ afirmam que um cliente se sente satisfeito quando sua percepção, experiência e os resultados do serviço atendem as suas expectativas.

Machado et al. ${ }^{12}$ avaliaram 376 pacientes e encontraram um índice de satisfação geral de $75,5 \%$ quanto ao tratamento recebido, afirmando que estes indicariam os serviços a terceiros. Já Suda et al. ${ }^{16}$, que avaliaram 51 pacientes de uma clínica escola, encontraram três quartos dos participantes relatando que se sentiram confortáveis na clínica, para retornar ou mesmo indicá-la a familiares e amigos, e sugerem que os dados são reflexo de uma visão humanística, crítica e profissional por parte da equipe de estagiários e supervisores.

O estudo de Teles et al. ${ }^{17}$ comparou a satisfação dos pacientes divididos em dois grupos, infantil (GI) e adulto (GA), e demonstrou um baixo índice percentual no quesito explicações oferecidas e segurança por parte do terapeuta, sendo avaliado como péssimo pelo GI. Os autores sugerem que a grande quantidade de pacientes atendidos, a proporção de profissionais, a disponibilidade e o cuidado do terapeuta ou os equipamentos disponíveis podem ser fatores a influenciar nos seus resultados.

No aspecto de ambiente físico, a pontuação foi maior quando comparada à de Moreira et al. ${ }^{11}$, relevando a informação de que existe diferença entre uma instituição pública e particular quanto ao dispêndio de investimento para os aspectos estruturais e materiais. O ambiente físico mais adequado eleva a percepção de qualidade do serviço pelo usuário.

Para Zeithami et al. ${ }^{18}$ os fatores situacionais influenciam o modo de percepção da qualidade do serviço. A sensação de dor e de prazer são aspectos influenciadores dessa percepção ${ }^{19}$. A satisfação dos pacientes, segundo Telles et al. ${ }^{17}$, está diversas vezes ligada à qualidade de vida, assim como a qualidade de vida está ligada à expectativa de vida, sendo que a expectativa pode variar de acordo com a idade, sexo, condição econômica, condição física e dor.

As doenças crônicas respiratórias e cardiovasculares estão altamente ligadas a quadros de depressão e de ansiedade, podendo ser causadas pelo aparecimento de sintomas limitantes das Atividades da Vida Diária, e ao risco de morte. A relação entre estresse, depressão e funcionalidade parece fazer parte de um complexo mecanismo que pode interferir na qualidade de vida ${ }^{20}$. O estudo de Santos e Faro ${ }^{21}$ sugere que o sentido de vida se associa diretamente com a autoestima e a satisfação com a vida, por isso a avaliação da qualidade de vida no momento da entrevista é um fator que pode diretamente refletir nos resultados encontrados, já que o aspecto emocional foi o de menor percepção da qualidade de vida.

$O$ aspecto social, entretanto, foi o de maior pontuação em relação à qualidade de vida, tendo como fundamento o conhecido papel que um programa de reabilitação cardiopulmonar pode gerar sobre o componente social, pois amplia a rede de suporte social. Em uma recente revisão sistemática que buscou descobrir as perspectivas dos pacientes de participação no exercício após um evento cardíaco, identificou-se que os domínios cognitivos e sociais foram os fatores mais citados que influenciam a participação em programas de exercícios ${ }^{22}$.

\section{CONCLUSÕES}

$\mathrm{P}$ or meio deste estudo, pôde-se comprovar que o serviço de fisioterapia desenvolvido pelo setor de cardiorrespiratória obteve bons resultados na avaliação de satisfação do paciente, assim como identificou que o aspecto social é o mais envolvido na percepção da qualidade de vida.

O estudo teve algumas limitações que poderiam trazer resultados mais detalhados caso fossem adotadas algumas melhorias, como avaliação de pré e pós-tratamento, implantação de análise por faixa etária. 


\section{Referências}

1. Ferreira LL, Marino LHC, Cavenaghi S. Fisioterapia cardiorrespiratória no paciente cardiopata. Rev Bras Clin Med. 2012; 10(2):127-131.

2. Ovando AC, Michaelsen SM, Dias JÁ, Herber V. Treinamento de marcha, cardiorrespiratório e muscular após acidente vascular encefálico: estratégias, dosagens e desfechos. Fisioter Mov. 2010; 23(3):253-269.

3. Karam I, Adawi T, Oldridge NB, Tarima SS, Stason WB, Shepard DS. Cardiac Rehabilitation Patient and Organizational Factors: What keeps patients in programs? J Am Heart Assoc. 2013; 2(5):e000418.

4. Lau HMC, Yin-Fat G, Jones AY, Lee EW, Siu EH, Hui DS. A randomised controlled trial of the effectiveness of an exercise training program in patients recovering from severe acute respiratory syndrome. Aust $\mathrm{J}$ Physiother. 2005; 51(4):213-219.

5. Guimarães FAB, Gardenghi G, Silva FMF. Reabilitação Cardíaca, tratamento e prevenção: Revisão Bibliográfica. Revista Movimenta. 2015; 8(1):50-59.

6. Domingues PW, Almeida AF. Fisioterapia como tratamento em portadores de doenças respiratórias obstrutivas. Saúde e Pesqui. 2010; 3(2):173-179.

7. Castro RRT, Negrão CE, Stein R, Serra SM, Teixeira JAC, Carvalho T, Araújo CGS, Alves MJNN. Diretriz de Reabilitação Cardíaca. Arq Bras Cardiol. 2005; 84(5):431-440.

8. Ries AL, Bauldoff GS, Carlin BW, Casaburi R, Emery CF, Mahler DA, Make B, Rochester CL, Wallack RZ, Herrerias C. Pulmonary Rehabilitation. Chest 2007; 131:4s-42s.

9. Rebello FPV, Garcia AS, Andrade DF, Werner CR, Carvalho T. Resultado clínico e econômico de um programa de reabilitação cardiopulmonar e metabólica. Arq Bras Cardiol. 2007; 88(3):321-328.

10. Souza CA, Santos RZ, Lineburger AA, Benetti M. Reabilitação cardiopulmonar e metabólica na atenção primária em saúde: é possível? R Bras Ci e Mov. 2015; 23(1):164-171.

11. Moreira CF, Borba JAM, Mendonça KMPP. Instrumento para aferir a satisfação do paciente com a assistência fisioterapêutica na rede pública de saúde. Fisioter Pesqui. 2007; 14(3):37-43.

12. Machado NP, Nogueira LT. Avaliação da satisfação dos usuários de serviços de Fisioterapia. Rev Bras Fisioter. 2008; 12(5):401-8.

13. Ciconelli RM, Ferraz MB, Santos W, Meinão I, Quaresma MR. Tradução para a língua portuguesa e validação do questionário genérico de avaliação de qualidade de vida SF-36 (Brasil SF-36). Rev Bras Reumatol. 1997; 39(3):143-50.

14. Silva CAS, Ferreira CES. Efeitos de um programa de reabilitação cardíaca na qualidade de vida relacionada á saúde (SF-36). Educação Física em Revista. 2011; 5(1):1-8.

15. Johnston R; Clark G. Administração de Operações de Serviços. 1. ed. 4. reimpr. São Paulo: Atlas, 2010.

16. Suda EY, Uemura MD, Velasco E. Avaliação da satisfação dos pacientes atendidos em uma clínica-escola de Fisioterapia de Santo André, SP. Fisioter Pesqui. 2009; 16(2):126-31.

17. Telles CM, Werneck MS, Marinho MP, Kanashiro MS, Braga DM. Satisfação do paciente na fisioterapia aquática. Caderno de Pós-Graduação em Distúrbios do Desenvolvimento. 2014; 14(1):41-52.

18. ZeithamI VA, Bitner MJ, Gremler DD. Marketing de serviços: a empresa com foco no cliente. 6 ed.

Bookman, 2014.

19. Mayer VF, Avila MG. A influência da estruturação da mensagem em comportamentos relacionados à saúde: um teste experimental. Saude Soc. 2010; 19(3):685-697.

20. Homann D, Stefanello JMF, Góes SM, Breda CA, Paiva ES, Leite N. Percepção de estresse e sintomas depressivos: funcionalidade e impacto na qualidade de vida em mulheres com fibromialgia. Rev Bras Reumatol. 2012; 52(3):319-330.

21. Santos LCS, Faro A. Relações entre autoestima e sentido de vida: Estudo com amostragem domiciliar em Aracaju (SE). Clínica \& Cultura. 2015; 4:54-69.

22. Campkin LM, Boyd JM, Campbell DJT. Coronary Artery Disease Patient Perspectives on Exercise Participation. J Cardiopulm Rehabil Prev. 2017; 37(5):305-314. 